\title{
Comparative Evaluation of Antibacterial Efficacy of Three Intracanal Medicaments in Primary Endodontic Infections: A Randomized Clinical Trial
}

\author{
Rakesh Mittal ${ }^{1}$, Monika Tandan $^{2}$, Suchita Sukul ${ }^{3}$
}

\begin{abstract}
Aim: To compare and evaluate the antibacterial efficacy of calcium hydroxide $(\mathrm{CH})$ paste, triple antibiotic paste (TAP), and double antibiotic paste (DAP).

Materials and methods: Ninety patients with ninety single-rooted teeth were selected. After complete disinfection, access opening was made and sample S1 was collected with paper point after injecting $5 \mathrm{~mL}$ of normal saline. After working length determination and biomechanical preparation till \#40 K-file, sample S2 was collected in the same manner as S1. The canals were dried and the three intracanal medicaments were mixed with propylene glycol. The patients were randomly divided into three groups $(n=30)$ and the medicaments (group I-CH, group II-TAP, and group III-DAP) were placed. After 7 days, following medicament removal, sample S3 was collected. Microbiological samples (S1, S2, and S3) were preincubated for 30 minutes and then plated on brain heart infusion agar and colonies were counted after 24 hours using the classic bacterial counting method.

Results: There was significant reduction of bacterial count from stages $\mathrm{S} 1$ to $\mathrm{S} 2$ and $\mathrm{S} 2$ to $\mathrm{S} 3$ in all the three experimental groups. The maximum reduction of bacterial count $\left(\mathrm{CFU} / \mathrm{mL} \times 10^{5}\right)$ at $\mathrm{S} 1, \mathrm{~S} 2$, and $\mathrm{S} 3$ stages and reduction in mean was seen in group III. However, there were no statistically significant difference between group II (TAP) and group III (DAP).
\end{abstract}

Conclusion: The TAP and DAP have similar antimicrobial efficacy though better than $\mathrm{CH}$.

Keywords: Antibacterial efficacy, Intracanal medicament, Minocycline.

Conservative Dentistry and Endodontic Journal (2020): 10.5005/jp-journals-10048-0056

\section{INTRODUCTION}

Microorganisms, especially bacteria and their toxins in the pulp, are able to induce pulpal inflammation and often lead to pulpal death. ${ }^{1}$ The aim of endodontic therapy is to eliminate microorganisms from the root canal system by an effective debridement and disinfection process. ${ }^{2}$ Regardless of the files used, technique of instrumentation, and type of irrigant used, antisepsis obtained from biomechanical preparation is temporary and partial. ${ }^{3}$

Consequently, chemical irrigants and intracanal medicaments seem necessary for eradication of infected tissues and microorganisms in addition to mechanical debridement. ${ }^{4}$ Various intracanal medicaments are advocated to eliminate bacteria and prevent multiplication of bacteria in-between appointments like ledermix, iodine potassium iodide, calcium hydroxide $(\mathrm{CH})$, chlorhexidine gel, triple antibiotic paste (TAP), double antibiotic paste (DAP), etc. ${ }^{5}$

Since its introduction in 1920, CH has been widely used in endodontics. It is a strong alkaline substance $(\mathrm{pH}=12.5)$. In an aqueous solution, it dissociates into calcium and hydroxyl ions. Various biological properties have been attributed to this substance, such as antimicrobial activity, tissue-dissolving ability, inhibition of tooth resorption, and induction of repair by hard tissue formation. Currently, it is acknowledged as one of the most effective antimicrobial dressings during endodontic therapy. ${ }^{6}$

Application of $\mathrm{CH}$ for a week has been shown to reduce the microorganisms. ${ }^{7}$ However, the buffering action of dentin neutralizes its action at deeper layers of dentinal tubules resulting in the survival of microorganisms. ${ }^{8}$ Enterococcus faecalis and Candida
${ }^{1-3}$ Department of Conservative Dentistry and Endodontics, Sudha Rustagi College of Dental Sciences and Research, Faridabad, Haryana, India

Corresponding Author: Suchita Sukul, Department of Conservative Dentistry and Endodontics, Sudha Rustagi College of Dental Sciences and Research, Faridabad, Haryana, India, Phone: +91 9643277909, e-mail: suchitashukul@gmail.com

How to cite this article: Mittal R, Tandan M, Sukul S. Comparative Evaluation of Antibacterial Efficacy of Three Intracanal Medicaments in Primary Endodontic Infections: A Randomized Clinical Trial. Cons Dent Endod J 2020;5(1):5-10.

Source of support: Nil

Conflict of interest: None

albicans have been reported to be resistant to the antimicrobial effect of $\mathrm{CH}$ due to their ability to penetrate the dentinal tubules and adapt to the changing environment. ${ }^{9-11}$

Antibiotics can be used as an adjunct to endodontic treatment, and its limited effectiveness through the systemic route of administration has led to their intracanal application to increase efficacy. Local application of antibiotics in the root canal has been suggested to overcome the potential risk of adverse systemic effects of antibiotics and as an effective mode for drug delivery in teeth lacking blood supply due to necrotic pulps or pulpless status. ${ }^{12}$

Because root canal infections are polymicrobial consisting of both aerobic and anaerobic bacterial species, single antibiotic may not be effective in canal disinfection. ${ }^{12}$ Several studies have reported the antimicrobial efficacy of TAP containing ciprofloxacin, 
metronidazole, and minocycline against the pathogens commonly found inside the root canal system. ${ }^{13}$

Triple antibiotic paste was introduced by Hoshino et al. in 1996 and contains three antibiotics that are ciprofloxacin, metronidazole, and minocycline dispensed together in a ratio of 1:1:1 and forms a paste when mixed with propylene glycol. ${ }^{14}$ Staining of the dentin by minocycline, a derivative of tetracycline, has been reported. Therefore, some authors suggest eliminating minocycline and keeping only metronidazole and ciprofloxacin in the antibiotic paste, which is known as DAP. ${ }^{15}$ Double antibiotic paste, a combination of metronidazole and ciprofloxacin, has been considered to possess antibacterial properties comparable to those of TAP and to minimize discoloration during endodontic regeneration. ${ }^{16}$ Therefore, the aim of this study was to comparatively evaluate the antibacterial efficacy of $\mathrm{CH}$, TAP, and DAP. The null hypothesis tested was that there would be no difference among antibacterial efficacy of three intracanal medicaments.

\section{Materials and Methods}

The present study was a randomized triple blinding parallel controlled clinical trial with balanced/equal randomization performed on 90 patients of age group 15-50 years at the Department of Conservative Dentistry and Endodontics, from June 2015 to June 2016. The allocation ratio was 1:1 and no dropouts were seen during the trial.

\section{Ethical Consideration}

The study protocol was approved by Institutional Ethical Committee of Sudha Rustagi College of Dental Sciences and Research. A written informed consent was obtained from each patient after recruiting them for the study.

\section{Inclusion Criteria}

Patients with noncontributory medical history, permanent teeth without any previous restoration, with a necrotic or infected pulp as diagnosed clinically and radiographically, with adequate coronal structure for proper isolation, temporization, and restoration.

\section{Exclusion Criteria}

Patients with systemic conditions, allergic to ciprofloxacin, metronidazole, and minocycline, acute periapical abscess, retreatment cases, patients on antibiotic therapy within 3 months, teeth with calcified canals, sinus opening, immature apex, internal or external resorption or periodontal pockets $>5 \mathrm{~mm}$, and pregnant women.

\section{Sample Size Calculation}

Based on the results of the pilot study and in order to obtain confidence interval level of 0.95 , at least $80 \%$ power, Open Epi Software version 3.01 was used for sample size estimation. It was decided to be 90 (each group-30).

\section{Randomization}

All the dispensed bottles of intracanal medicaments were covered with black papers and numbered from 1 to 90 . Subjects were randomly assigned with the help of computer-generated random number list to one of three parallel groups in 1:1:1 ratio. They were randomly allocated to one of the groups: $\mathrm{CH}$, TAP, or DAP. All the dispensed bottles of $\mathrm{CH}$, TAP, or DAP were numbered from 1 to 30,31 to 60 , and 61 to 90, respectively. Each subject was assigned an order number (using random number list) and handed over by the same number prepacked bottle. This was done to prevent any contamination bias and to ensure blinding. Hence, the examiner, patient, and investigator were not aware of the groups. Help of an intern was taken to ensure smooth functioning of the trial (Flowchart 1).

\section{Intervention}

Each tooth was anesthetized (Biocaine-ADR Local Anesthetic solution, Biochem Pharmaceutical Industries Ltd., India) and isolated with rubber dam (Hygenic Dental Dam Kit, Coltene Whaledent, Switzerland) followed by caries removal. The oral cavity along with the experimental tooth were disinfected with the betadine solution (Povidone lodine Solution, Abbott Healthcare Pvt. Ltd., India). To ensure thorough disinfection, a disinfection sample (S0) was taken by flooding the tooth with $0.9 \%$ normal saline and absorbing the contents with paper points (Metabiomed, India). The access cavity was prepared using a high-speed endo access bur \#2 (DentsplyMaillefer, Switzerland) under water spray and the working length was determined radiographically. Pretreatment sample (S1) was obtained by injecting $5 \mathrm{~mL}$ of normal saline $(0.9 \% \mathrm{v} / \mathrm{w}$, Lifusion, India) into the root canal and circumferentially pumping a \#10 K-file (1 $\mathrm{mm}$ short of working length). A sterile paper point was placed into the canal for 60 seconds. It was then immediately transported to the test tube containing transport media (Peptone water, HiMedia Laboratories Pvt. Ltd., India). Three samples were taken for each tooth. Biomechanical preparation was done using the step back technique up to master apical size \#40 K-file. Saline as an irrigant was used followed by collection of the post-instrumentation sample (S2) in the same manner as S1.

The patients were divided randomly into three experimental groups:

- Group I: CH was mixed with propylene glycol.

- Group II: One tablet each of ciprofloxacin (Ciplox 500 mg, Cipla, India), metronidazole (Metrogyl 400 mg, J. B. Chemicals and

Flowchart 1: CONSORT 2010 flow diagram

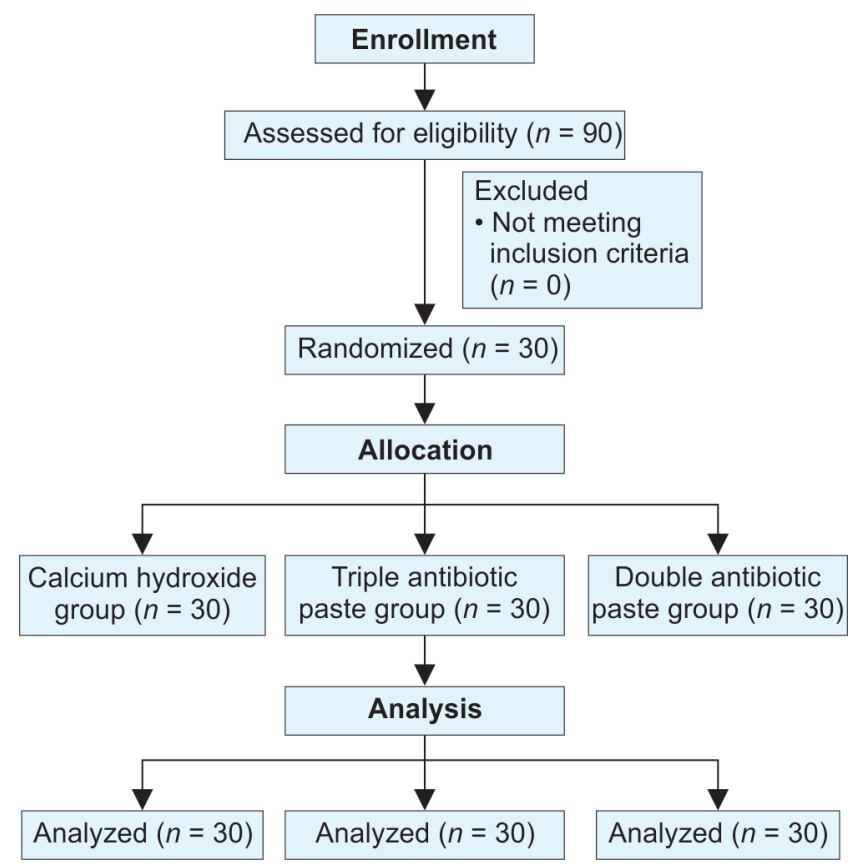


Pharmaceuticals Ltd., India), and minocycline (Minoz 100 mg, Cipla, India) (1:1:1) were taken and powdered finely by using mortar pestle. The powder was then mixed with propylene glycol.

- Group III: One tablet each of ciprofloxacin (Ciplox 500 mg, Cipla, India) and metronidazole (Metrogyl $400 \mathrm{mg}$, J. B. Chemicals and Pharmaceuticals Ltd., India) (1:1) were taken and powdered by using mortar pestle. Then they were mixed with propylene glycol in the similar manner as in group II.

The medicaments were sterilized and then placed in the canal using lentulospiral. Extent and compactness of the intracanal medicament were checked with intraoral periapical radiovisiography (RVG). Double seal of Cavit G (Orafil-G, PREVEST DenPro, India) was placed. After 7 days, medicament was removed by thorough irrigation with normal saline. The root canal was checked for residual medicament with the paper point. Post-medication sample (S3) was collected in the same manner as S2. Teeth were obturated by the lateral condensation technique using \#40 gutta-percha (Metabiomed, India) and AH Plus sealer (Dentsply, Germany). The teeth were then restored with composite (Spectrum, Dentsply India Pvt. Ltd., India).

\section{Microbiological Procedure}

Microbiological samples (S1, S2, S3) were preincubated for 30 minutes and shaken vigorously in a vortex mixture for 60 seconds and then were plated on Brain heart infusion agar (HiMedia Laboratories Pvt. Ltd., India) and colonies were counted after 24 hours using the classic bacterial counting method on Digital Colony Counter (HiMedia Laboratories Pvt. Ltd., India).

\section{Outcome Measures}

The primary outcome measures used for this study were colonyforming units $/ \mathrm{mL}(\mathrm{CFU} / \mathrm{mL})$ count of S1, S2 at Day 1, and S3 after 7 days. Any adverse or unintended effects of the intervention were taken as secondary outcome measures.

\section{Statistical Analysis}

The data were entered into the Excel sheet (MS Office 2007) and then analyzed using the SPSS software 21 version (Statistical Package for Social Sciences). Summarized data were presented using tables and graphs. Data were normally distributed as tested using the ShapiroWilk W test ( $p$ value was more than 0.05). Therefore, analysis was performed using the parametric tests, i.e., independent $t$-test (for comparing two groups) and one-way ANOVA test (for comparing more than two groups). The level of statistical significance was set at $p$ value less than 0.05 . The post hoc Tukey's test was used for pairwise comparison of subgroups (ANOVA test is positive, i.e., $p$ is less than the selected significance level).

\section{Results}

The findings of this study show that there was significant reduction of bacterial count from stages S1 to S2 and S3 in all the three experimental groups. The maximum reduction of bacterial count $\left(\mathrm{CFU} / \mathrm{mL} \times 10^{5}\right)$ at $\mathrm{S} 1, \mathrm{~S} 2$, and S3 stages and reduction in mean were seen in group III (DAP). Also antimicrobial activity of TAP and DAP was significantly greater than the antimicrobial activity of $\mathrm{CH}$ (Tables 1 to 3 ).

Table 3 shows comparison of antibacterial efficacy of three intracanal medicaments. On applying the Tukey's post hoc test (Table 2) to compare the antimicrobial activity of three intracanal medicaments, TAP and DAP showed significantly greater
Table 1: Mean of reduction of bacterial count at stages S1, S2, and S3 for $\mathrm{CH}$ paste, antibiotic paste with minocycline, and antibiotic paste without minocycline

\begin{tabular}{|c|c|c|c|c|}
\hline \multicolumn{5}{|c|}{ Mean reduction values } \\
\hline & & Group / CH & Group II TAP & $\begin{array}{l}\text { Group III } \\
\text { DAP }\end{array}$ \\
\hline \multirow[t]{3}{*}{ Mean } & S1-S2 & 1.29 & 2.12 & 2.17 \\
\hline & S1-S3 & 1.30 & 1.03 & 1.44 \\
\hline & $\mathrm{S} 2-\mathrm{S} 3$ & 0.61 & 1.09 & 0.73 \\
\hline \multirow{3}{*}{$\begin{array}{l}\text { Standard } \\
\text { deviation }\end{array}$} & $\mathrm{S} 1-\mathrm{S} 2$ & 0.79 & 0.79 & 0.75 \\
\hline & S1-S3 & 0.80 & 0.63 & 0.72 \\
\hline & S2-S3 & 0.29 & 0.53 & 0.26 \\
\hline$p$ value & $\begin{array}{l}\mathrm{S} 1-\mathrm{S} 2 / \mathrm{S} 1- \\
\mathrm{S} 3 / \mathrm{S} 2-\mathrm{S} 3\end{array}$ & $<0.05^{*}$ & $<0.05^{*}$ & $<0.05^{*}$ \\
\hline
\end{tabular}

aPaired $t$-test

*Significance of relationship at $p<0.05$

Table 2: Post hoc analysis

\begin{tabular}{llll}
\hline$p$ value & Group I (CH) & Group II (TAP) & Group III (DAP) \\
\hline Group I (CH) & - & $0.00^{*}$ & $0.001^{*}$ \\
Group II (TAP) & - & - & 0.231 \\
Group III (DAP) & - & - & - \\
\hline
\end{tabular}

*Significance of relationship at $p<0.05$

antimicrobial activity as compared to $\mathrm{CH}$. But no significant difference was seen in the antimicrobial activity of TAP and DAP.

\section{Discussion}

Primary endodontic infections are dominated by obligate anaerobes, whereas facultative anaerobes and Candida spp. are frequently encountered in secondary endodontic infections. ${ }^{17}$ The use of a biocompatible intracanal medicament with antimicrobial properties between appointments may completely eradicate the microorganisms from the root canal system and could significantly increase the success of root canal treatment. ${ }^{18}$

Recontamination of the root canal will only occur by medicament solubilization by saliva; medicament permeability to saliva; and percolation of saliva at the interface between the medicament and the root canal walls. However, the root canal system can become recontaminated when the number of bacterial cells exceeds the antibacterial activity of the medicament. ${ }^{6}$

Several studies have vouched to the ineffectiveness of $\mathrm{CH}$ in eliminating bacterial cells completely. Haapasalo and Orstavik reported that $\mathrm{CH}$ failed to eliminate, even superficially, E. faecalis in dentinal tubules. ${ }^{19}$ Safavi et al. demonstrated that E. faecium remained viable in dentinal tubules after relatively extended periods of $\mathrm{CH} /$ saline mixture treatment. ${ }^{20}$ Orstavik and Haapasalo stated that $\mathrm{CH}$ could take up to 10 days to disinfect dentinal tubules infected by facultative bacteria. ${ }^{21}$ Also, Estrela et al. observed that $\mathrm{CH}$ in infected dentinal tubules had no antimicrobial effect on $S$. faecalis, S. aureus, B. subtilis, P. aeruginosa, or on the bacterial mixture used throughout the experiment. ${ }^{22}$

Antibiotics are used in dentistry both systemically and topically. During systemic administration of antibiotics, negligible concentrations reach the root canal, whereas during the local administration of antibiotics, greater concentrations can be achieved when used as intracanal medicaments. Combination of irrigants or medicaments decreases the development of resistant 
Efficacy of Intracanal Medicaments in Endodontic Infections

Table 3: Comparison of antibacterial efficacy of three intracanal medicaments at stages S2-S3

\begin{tabular}{llllll}
\hline Test dentifrice & Mean & Standard deviation & Fvalue & $p$ value & Tukey's post hoc test \\
\hline CH & 0.61 & 0.29 & 8.59 & $0.00^{*}$ & $2>1, \mathrm{~S}$ \\
TAP & 1.09 & 0.53 & & & $3>1, \mathrm{~S}$ \\
DAP & 0.73 & 0.26 & & & $2>3, \mathrm{NS}$ \\
\hline
\end{tabular}

One-way ANOVA test, ${ }^{*}$, significant; NS, nonsignificant

bacterial strains and produces the synergistic effect, whose antimicrobial action lasts longer and also results in sustained release of medicaments. ${ }^{23}$

Triple antibiotic paste can penetrate into dentinal tubules and has proved to be an effective antimicrobial agent. ${ }^{18}$ Metronidazole has a broad spectrum of activity against anaerobic bacteria and protozoa. Minocycline is bacteriostatic and has a wide range of activity against gram-positive and gram-negative bacteria through prevention of protein synthesis by the organism. Ciprofloxacin has a rapid bactericidal activity and is more effective against gramnegative bacteria. ${ }^{24} \mathrm{Adl}$ et al. showed that metronidazole is the most effective component of TAP against $E$. faecalis. ${ }^{25}$ Research with topical antibiotics has shown that a combination of metronidazole, ciprofloxacin, and minocycline is effective in killing common endodontic pathogens from necrotic/infected root canals in vitro as well as proved to be an effective disinfectant in vivo. Furthermore, the TAP has been used successfully in regenerative endodontic treatments and in healing of large periradicular lesions. ${ }^{26}$ It promotes healing, repairs periapical tissue besides creating an aseptic environment and accelerates functional development of the pulp-dentin complex. ${ }^{18} \mathrm{Adl}$ et al. stated in their study that in comparison with $\mathrm{CH}$, TAP significantly decrease the number of CFUs in 100 and $200 \mu \mathrm{m}$ dentinal depths on day 1 and 7 application, thus proving TAP is more efficacious against $E$. faecalis. ${ }^{13}$ Pai et al. stated in their study that $\mathrm{CH}$ and TAP are effective in managing interappointment flare-ups in diabetic patients. ${ }^{12}$

However, care should be taken in patients who are known to be allergic to tetracycline, and also in anterior teeth where discoloration could be a side effect caused by minocycline. ${ }^{18} \mathrm{Kim}$ et al. identified minocycline as the cause for discoloration in vitro. ${ }^{27}$ It is thought to bind with the calcium of dentin forming insoluble complexes, resulting in discoloration. ${ }^{28}$

To overcome the disadvantage of discoloration with TAP, DAP, which is a combination of only metronidazole and ciprofloxacin, has been used successfully in endodontic regeneration and was suggested as a substitute for TAP as an intracanal medicament. ${ }^{15}$

Sabrah et al. reported that TAP and DAP showed a significant residual antibacterial effect in human radicular dentine when treated with various concentrations of TAP and DAP. All concentrations of DAP showed a significantly longer residual antibacterial effect when compared with the similar concentrations of TAP. ${ }^{29}$ Also, Ferreira et al. stated that TAP and DAP have better antibacterial efficacy as intracanal medicaments against $E$. faecalis in necrotic immature teeth and remained active for 30 days. ${ }^{30}$

Although TAP and DAP have better antimicrobial effect than $\mathrm{CH}$, there are some studies which reported that $\mathrm{CH}$ is more biocompatible to the human periodontal ligament (HPDL) cells and stem cell from apical papilla (SCAP) cells, which are responsible for regenerative procedures in endodontics. According to Yadlapati et al. who demonstrated their results that HPDL cells treated by $\mathrm{CH}$ had a viability greater than the $70 \%$ survival cut-off recommended by the international standards for the evaluation of medical materials.
These results corroborate the findings where $\mathrm{CH}$ also promoted the best results regarding biocompatibility on SCAP survival and proliferation. With regard to the antibiotic pastes, the results for TAP showed that this material is highly cytotoxic to HPDL fibroblasts. ${ }^{31}$

Many in vitro studies have been done on the evaluation and comparison of antimicrobial efficacy of $\mathrm{CH}, \mathrm{TAP}$, and DAP but none had reported a randomized clinical trial. Most of the in vivo studies have been done for TAP or DAP on regenerative procedures in infected, open apex teeth but very few studies have been done on fully mature infected teeth. Therefore, this study was undertaken to comparatively evaluate the antimicrobial efficacy of three medicaments by counting the colonies formed after culturing the samples taken from the patients at different stages, i.e., S1, S2, and S3.

To reduce the variation in results, only asymptomatic teeth were included; teeth with acute clinical signs or with sinus tracts were excluded, as they have greater and variable bacterial load. Additionally, the periapical exudate seeping into the root canal between treatment sessions can result in increased bacterial counts at the second session. Teeth with coronal restoration were also excluded because the bacteria at the restoration margins can cause contamination during the medication period. ${ }^{17}$ Before starting the treatment, an informed consent was taken from each patient.

A negative control group with no medication was not included due to ethical concerns. Different medicaments were applied for 7 days so that their complete antimicrobial effect could be obtained as reported in previous studies. The presence of false-positive and negative cultures makes the results obtained from bacterial cultures questionable. False-positive results can occur because of salivary leakage, contamination during sampling, and clinical/ laboratory handling. To avoid this, sterility check sample (SO) were taken. False-negative results can occur due to carryover effect of the medicaments used during therapy, so they were removed as much as possible before acquiring the post-medication samples (S3) by thorough irrigation with normal saline. ${ }^{17}$ Transferring of any residual antimicrobial agent from the root canal to the transport medium and culture medium will provide the less CFUs/mL (due to antibacterial effect), which simulates the condition in root canal, as remaining medicament in the root canal will provide residual antibacterial effect of the intracanal medicament after completion of the root canal treatment.

According to Universal Protocol for Irrigation, sodium hypochlorite (5.25\%) should be used as an antimicrobial agent but it can make a difference in S2 and S3 samples with significant effect on the statistical analysis. Also, it can provide the false-negative results.

Calcium hydroxide is the most commonly utilized and studied root canal medication. ${ }^{32}$ Calcium hydroxide was used as a positive control. The pharmaceutical carrier propylene glycol was used to make a paste with TAP and DAP. This ingredient allows increased solubility and delivery of paste into the root canal. Aqueous solutions of antibiotics can often degrade and this degradation is increased by a rise in temperature and $\mathrm{pH} .{ }^{26} \mathrm{According}$ to $\mathrm{Chua}$ et al., $\mathrm{CH}$ with 
propylene glycol resulted in statistically significant inhibition of $C$. albicans at dentinal tubule depths of 200 and $400 \mu \mathrm{m}$ on day 1 and day 7. Calcium hydroxide was effective there, which probably is due to the addition of a vehicle, namely propylene glycol, which allows the release of hydroxyl ions for a longer period, and enhances the diffusibility of $\mathrm{CH}$ into the dentinal tubules. ${ }^{18}$

Sjogren et al. concluded that $\mathrm{CH}$ as short-term intracanal dressing after 7 days efficiently eliminated bacteria that survived biomechanical instrumentation of the canal, while the 10-minute application was ineffective. ${ }^{7}$ Therefore, in this study $\mathrm{CH}$ was placed in the root canal as medicament for 7 days.

Some general dental practitioners either use saline, local anesthetics, and/or distilled water to flush the canal before obtaining the samples from the root canal system. These have no antibacterial action and will not affect the antimicrobial activity of the medicament. These irrigants may be used frequently as they are easy to use and readily available. Therefore, samples were obtained after injecting normal saline $(5 \mathrm{~mL})(0.9 \% \mathrm{v} / \mathrm{w}$, Lifusion, India) into the root canal. ${ }^{33}$

The results of this study demonstrated that there was significant reduction of bacterial count from stages S1 to S2 and S2 to S3 in all the three experimental groups (intracanal medicaments). The maximum reduction of bacterial count $\left(\mathrm{CFU} / \mathrm{mL} \times 10^{5}\right)$ at $\mathrm{S} 1, \mathrm{~S} 2$, and S3 stages and reduction in mean were seen in group III. Also antimicrobial activity of group II and group III was significantly greater than the antimicrobial activity of group I. No significant difference was observed between the mean reduction of group I and group II. These results are in accordance with the other studies. According to Maniglia et al. TAP was significantly more effective against $E$. faecalis, and showed the largest halo of inhibition followed by DAP, with said inhibition remaining stable throughout the 30-day period. In contrast, $\mathrm{CH}$ group along with saline and chlorhexidine, no antibacterial effect was observed. ${ }^{30}$

Sabrah et al. investigated the effect of various dilutions of TAP and DAP used in endodontic regeneration on the survival of human dental pulp stem cells (DPSCs) and also determined their antibacterial effect against established $E$. faecalis biofilm. All tested dilutions had an antibacterial effect against $E$. faecalis. However, $0.125 \mathrm{mg} / \mathrm{mL}$ of DAP and TAP showed a significant antibacterial effect with no cytotoxic effects on DPSCs. ${ }^{29}$ Therefore, it was concluded that when similar antimicrobial efficacy can be seen from the previous studies, the choice of intracanal medicament should be DAP.

\section{CONCLUSION}

Antimicrobial activity of TAP and DAP was significantly greater than $\mathrm{CH}$ when used as intracanal medicament during root canal treatment. Also, TAP and DAP had similar antimicrobial activity.

\section{References}

1. Chuensombat S, Khemaleelakul S, Chattipakorn S, et al. Cytotoxic effects and antibacterial efficacy of a 3-antibiotic combination: An in vitro study. J Endod 2013;39(6):813-819. DOI: 10.1016/ j.joen.2012.11.041.

2. Agrafioti A, Tzimpoulas NE, Kontakiotis EG. Influence of dentin from the root canal walls and the pulp chamber floor on the $\mathrm{pH}$ of intracanal medicaments. J Endod 2013;39(5):701-703. DOI: 10.1016/ j.joen.2012.12.039.

3. Dealmeidagomes B, Ferraz C, Garrido F, et al. Microbial susceptibility to calcium hydroxide pastes and their vehicles. J Endod 2002;28(11): 758-7561. DOI: 10.1097/00004770-200211000-00003.
4. Mozayeni MA, Haeri A, Dianat $\mathrm{O}$, et al. Antimicrobial effects of four intracanal medicaments on Enterococcus faecalis: an in vitro study. Iran Endod J 2014;9(3):195-198.

5. Athanassiadis B, Abbott PV, Walsh LJ. The use of calcium hydroxide, antibiotics and biocides as antimicrobial medicaments in endodontics. Aust Dent J 2007;52(1 Suppl):S64-S82. DOI: 10.1111/ j.1834-7819.2007.tb00527.x.

6. Siqueira JFJr, Lopes HP. Mechanisms of antimicrobial activity of calcium hydroxide: a critical review. Int Endod J 1999;32(5):361-369. DOI: 10.1046/j.1365-2591.1999.00275.x.

7. Sjogren U, Figdor D, Spångberg L, et al. The antimicrobial effect of calcium hydroxide as a short-term intracanal dressing. IntEndod J 1991;24(3):119-125. DOI: 10.1111/j.1365-2591.1991.tb00117.x.

8. Madhubala MM, Srinivasan N, Ahamed S. Comparative evaluation of propolis and triantibiotic mixture as an intracanal medicament against Enterococcus faecalis. J Endod 2011;37(9):1287-1289. DOI: 10.1016/j.joen.2011.05.028.

9. Evans M, Davies JK, Sundqvist G, et al. Mechanisms involved in the resistance of Enterococcus faecalis to calcium hydroxide. Int Endod J 2002;35(3):221-228. DOI: 10.1046/j.1365-2591.2002.00504.x.

10. Mozayeni MA, Hadian A, Bakhshaei P, et al. Comparison of antifungal activity of $2 \%$ chlorhexidine, calcium hydroxide, and nanosilver gels against Candida albicans. J Dent (Tehran) 2015;12(2):109-117.

11. Waltimo TM, Siren EK, Orstavik D, et al. Susceptibility of oral Candida species to calcium hydroxide in vitro. Int Endod J 1999;32(2):94-98. DOI: 10.1046/j.1365-2591.1999.00195.x.

12. Pai S, Pai ARV, Thomas MS, et al. Effect of calcium hydroxide and triple antibiotic paste as intracanal medicaments on the incidence of inter-appointment flare-up in diabetic patients: an in vivo study. J Conserv Dent 2014;17(3):208-211. DOI: 10.4103/0972-0707. 131776.

13. Adl A, Hamedi S, Shams MS, et al. The ability of triple antibiotic paste and calcium hydroxide in disinfection of dentinal tubules. Iran Endod J 2014;9(2):123-126.

14. Parasuraman VR, Muljibhai BS. 3Mix-MP in endodontics-an overview. Indian journal of dental and medical sciences 2012;3(1):36-45. DOI: 10.9790/0853-0313645.

15. Yassen GH, Chu TM, Eckert G, et al. Effect of medicaments used in endodontic regeneration technique on the chemical structure of human immature radicular dentin: an in vitro study. J Endod 2013;39(2):269-273. DOI: 10.1016/j.joen.2012.09.020.

16. Algarni AA, Yassen GH, Gregory RL. Inhibitory effect of gels loaded with a low concentration of antibiotics against biofilm formation by Enterococcus faecalis and Porphyromonas gingivalis. J Oral Sci 2015;57(3):213-218. DOI: 10.2334/josnusd.57.213.

17. Sinha N, Patil S, Dodwad PK, et al. Evaluation of antimicrobial efficacy of calcium hydroxide paste, chlorhexidine gel, and a combination of both as intracanal medicament: an in vivo comparative study. J Conserv Dent 2013;16(1):65-70. DOI: 10.4103/0972-0707.105302.

18. Chua EG, Parolia A, Ahlawat $P$, et al. Antifungal effectiveness of various intracanal medicaments against Candida albicans: an ex-vivo study. BMC Oral Health 2014;14(53):1-8. DOI: 10.1186/1472-6831-14-53.

19. Haapasalo $M$, Orstavik $D$. In vitro infection and disinfection of dentinal tubules. J Dent Res 1987;66(8):1375-1379. DOI: 10.1177/00220345870660081801.

20. Safavi KE, Spangberg L, Langeland K. Root canal dentinal tubule disinfection. J Endod 1990;16(5):207-210. DOI: 10.1016/s00992399(06)81670-5.

21. Orstavik D, Haapasalo M. Disinfection by endodontic irrigants and dressings of experimentally infected dentinal tubules. Endod Dent Traumatol 1990;6(4):142-149. DOI: 10.1111/j.1600-9657.1990. tb00409.x.

22. Estrela C, Pimenta FC, Ito IY, et al. Antimicrobial evaluation of calcium hydroxide in infected dentinal tubules. J Endod 1999;25(6):416-418. DOI: 10.1016/S0099-2399(99)80269-6.

23. Shaik J, Garlapati R, Nagesh B, et al. Comparative evaluation of antimicrobial efficacy of triple antibiotic paste and calcium hydroxide using chitosan as carrier against Candida albicans and Enterococcus 
faecalis: an in vitro study. J Conserv Dent 2014;17(4):335-339. DOI: 10.4103/0972-0707.136444.

24. Bazvand L, Aminozarbian MG, Farhad A, et al. Antibacterial effect of triantibiotic mixture, chlorhexidine gel, and two natural materials propolis and aloe vera against Enterococcus faecalis: an ex vivo study. Dent Res J (Isfahan) 2014;11(4):469-474.

25. Adl A, Shojaee NS, Motamedifar M. A comparison between the antimicrobial effects of triple antibiotic paste and calcium hydroxide against Enterococcus faecalis. Iran Endod J 2012;7(3):149-155.

26. Taneja S, Kumari M, Parkash H. Nonsurgical healing of large periradicular lesions using a triple antibiotic paste: a case series. ContempClin Dent 2010;1(1):31-35. DOI: 10.4103/0976-237X.62519.

27. Kim JH, Kim Y, Shin SJ, et al. Tooth discoloration of immature permanent incisor associated with triple antibiotic therapy: a case report. J Endod 2010;36(6):1086-1091. DOI: 10.1016/j.joen.2010.03.031.

28. Thomas MS. Crown discoloration due to the use of triple antibiotic paste as an endodontic intra-canal medicament. Saudi Endod J 2014;4(1):32-35. DOI: 10.4103/1658-5984.127985.
29. Sabrah AH, Yassen GH, Liu WC, et al. The effect of diluted triple and double antibiotic pastes on dental pulp stem cells and established enterococcus faecalis biofilm. Clin Oral Investig 2015;19(8):2059-2066. DOI: 10.1007/s00784-015-1423-6.

30. Maniglia-Ferreira C, de Almeida-Gomes F, Pinto MM, et al. In vitro evaluation of the antimicrobial effects of different intracanal medications in necrotic immature teeth. Eur Arch Paediatr Dent 2016;17(4):251-255. DOI: 10.1007/s40368-016 -0236-x.

31. Yadlapati M, Souza LC, Dorn S, et al. Deleterious effect of triple antibiotic paste on human periodontal ligament fibroblasts. Int Endod J 2014;47(8):769-775. DOI: 10.1111/iej.12216.

32. Gupta SP, Bhati M, Jhajharia K, et al. Evaluation of antimicrobial and antifungal efficacy of inter appointment intracanal medicaments against Enterococcus and Candida albicans: an in vitro study. J Int Oral Health 2015;7(6):97-102.

33. Iqbal A. Antimicrobial irrigants in the endodontic therapy. Int J Health Sci (Qassim) 2012;6(2):186-192. DOI: 10.12816/0005998. 